\title{
Supervivencia de mujeres jóvenes con cáncer de mama en el Instituto de Cancerología Las Américas entre 2007 y 2016.
}

\section{Survival of young women with breast cancer in a colombian referral cancer center between 2007-2016}

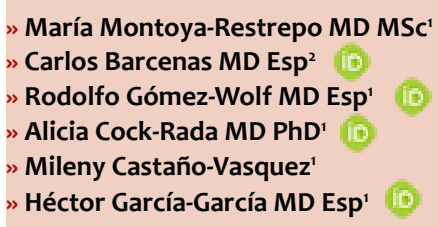

${ }^{1}$ Instituto de Cancerología (IDC) Las Américas auna, Medellín - Colombia.

${ }^{2}$ University of Texas MD Anderson Cancer Center.

Recibido el 15 de julio de 2020; Aceptado el 22 de agosto de 2020

\section{Resumen}

Antecedentes: La incidencia de Cáncer de Mama (CaMa) en jóvenes latinoamericanas es 20 \%, en Colombia fluctúa entre 10 y $24 \%$. Su gravedad se atribuye al diagnóstico en estadios avanzados, mayor frecuencia de tumores triple negativo y $\mathrm{HER} 2+$.

Objetivo: Describir la supervivencia global (SG) de mujeres $\leq 40$ años con CaMa infiltrante operadas en el Instituto de Cancerología Las Américas auna (Medellín-Colombia), entre 2007-2016.

Materiales y métodos: Estudio de seguimiento de una cohorte retrospectiva con datos del registro institucional. La supervivencia se calculó desde el diagnóstico hasta la fecha del último control médico o muerte. Se elaboraron curvas de supervivencia de Kaplan Meier y se compararon con log rank test.

Resultados: En el período se operaron 4.059 mujeres, de estas 390 (9.6 \%) tenían $\leq 40$ años, 365 pacientes cumplieron criterios de inclusión. Al diagnóstico 67 (18.3\%) estadio I, 152 (41.6\%) estadio II, 140 (38.3\%) estadio III y 6 (1.6\%) estadio IV. Por subtipos moleculares hubo 87 (24.1\%) Luminal A, 152 (42.1\%) Luminal B HER2-, 72 (19.9 \%) Luminal B HER2+, 17 (4.7 \%) HER2- enriquecido y 33 (9.1\%) Triple Negativo. $24.4 \%$ de pacientes fueron HER2-positivas. Tuvieron recaída loco-regional 34 (9.3 \%) pacientes y 132 (36.2\%) desarrollaron metástasis a distancia. La mediana de seguimiento fue 60 meses (rango 8-142 meses) y la SG de 81.3\% (IC95\%: 76.3-85.4). La SG de tumores triple negativo fue la más baja, 67.7\% (IC95 \%: 48-81.3).

Conclusiones: Se encontró mayor porcentaje de pacientes con cáncer Luminal B y HER2 positivo

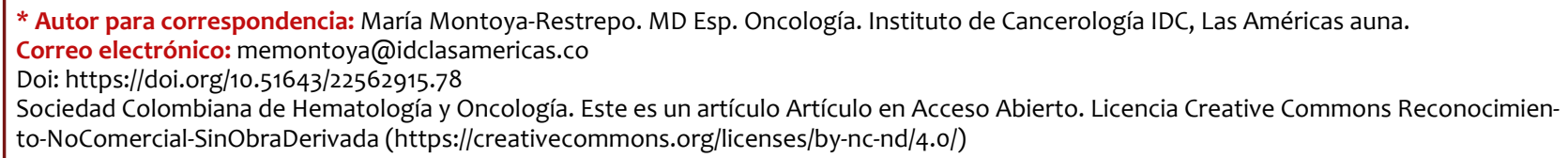


a lo reportado en la literatura. La SG fue similar a las reportadas por otros estudios y menor en estadios avanzados y tumores triple negativo.

Palabras clave: Neoplasias de la mama; salud de la mujer; análisis de sobrevivencia; Colombia.

\section{Abstract}

Background. The incidence of breast cancer in young women in Latin America is about 20\%, and in Colombia, it is around $10 \%$ to $24 \%$. Its aggressive behavior is likely due to advance stages at diagnosis and a major frequency of triple negative and Her2-positive subtypes.

Objective. This study describes the overall survival (OS) of women $\leq 40$ years old with infiltrating breast cancer that underwent breast surgery between 2007-2016 at the Institute de Cancerología Las Américas auna (Medellín-Colombia).

Materials and methods: This is a retrospective observational study. We performed a survival analysis using the Kaplan-Meier method and compared the survival curves using the Log-Rank test. We calculated OS from the date of diagnosis of breast cancer to the date of the last clinic visit or date of death. We compared our results to those reported by other countries in the literature.

Results. Of 4059 women who underwent breast surgery, 390 (9.6\%) were $\leq 40$ years-old, and 365 patients met inclusion criteria. At diagnosis 67 patients (18.3\%) were stage I, $152(41.6 \%)$ stage II, 140 (38.3\%) stage III and 6 (1.6\%) stage IV. According to the molecular intrinsic subtypes $87(24.1 \%)$ were Luminal A, 152 (42.1\%) Luminal B Her2-negative, 72 (19.9\%) Luminal B Her2-positive, 17 (4.7\%) Her2-enriched and 33 (9.1\%) Triple Negative. Loco-regional relapse was seen in 34 patients (9.3\%) and 132 (36.2\%) developed systemic disease. The median OS time was 60 months (range: 8-142 months), with an OS of $81.3 \%$ (IC95\%: 76.3-85.4). Patients who had Triple Negative disease had the lowest 5-year OS (67.7\%, IC95\%: 48-81.3).

Conclusions. Patient characteristics differ from what is reported in other countries, in the higher percentage of patients with Luminal B and Her2-positive tumors and the lower proportion of Triple Negative tumors. The OS according to the cancer staging and the molecular subtypes was similar to other countries.

Keywords: Breast neoplasms; women's health; survival analysis; Colombia

\section{Introducción}

La incidencia de cáncer de seno en mujeres jóvenes en Latinoamérica está alrededor del 20 $\%$, y en Colombia oscila entre 10 y $24 \%^{1-3}$. No hay consenso acerca del punto de corte para definir qué es una paciente joven, la mayoría de artículos se refieren a mujeres con edad menor a 350 40 años ${ }^{4}$. Algunos autores definen mujer joven como aquella que es premenopaúsica ${ }^{5}$.

La edad per se como factor pronóstico en cáncer de seno, es un hecho controvertido; su comportamiento agresivo podría deberse al diagnóstico en estadios avanzados, una mayor frecuencia de tumores triple negativo y HER2 positivo ${ }^{6,7}$. Las pacientes jóvenes son una población de especial interés, ya que en general reciben tratamientos más agresivos debido a su temprana edad y también tienen necesidades diferentes a las pacientes de mayor edad, ya que tienen necesidades a nivel de fertilidad y están en el auge de su carrera laboral.

En Colombia, no se conocen las características clínicas ni la supervivencia de las mujeres jóvenes con cáncer de seno. El objetivo de este estudio fue describir la supervivencia global 
(SG) de mujeres con edad $\leq 40$ años, con cáncer de seno infiltrante, llevadas a cirugía en el Instituto de Cancerología (IDC), entre el 2007 y el 2016.

\section{Materiales y métodos}

Este es un estudio observacional retrospectivo. Se revisaron las historias clínicas de mujeres con edad entre 18 y 40 años que recibieron manejo para cáncer de seno en el Instituto de Cancerología (IDC) Las Américas auna (MedeIlín-Colombia), con alguna de las siguientes terapias: radioterapia, cirugía, terapia hormonal o quimioterapia. Se excluyeron pacientes con segundos primarios (excepto cáncer de piel diferente al melanoma y la neoplasia cervical in situ) y quienes tuvieran historia clínica incompleta con menos del $25 \%$ de los datos.

La clasificación de los subtipos moleculares fue realizada de acuerdo a cuatro biomarcadores usando inmunohistoquímica (ICH): los receptores hormonales de estrógeno (ER) y de progesterona (PR) se consideraron positivos si la tinción por IHC era $\geq 1 \%$, el HER2- se consideró positivo si la ICH era de 3+ o FISH positivo (rango $\geq 2$ ) siguiendo las guías de ASCO/CAP ${ }^{8}$. Los tumores fueron clasificados en los siguientes subgrupos: Luminal A: ER+ y/o PR+, HERz-negativo, ki-67<14\%; Luminal $B: E R+y / o ~ P R+$, HER2-negativo, ki-67 $\geq 14 \%$; Luminal B/HER2+: $\mathrm{ER}+$ y/o PR+, HER2-positivo; HER2-enriquecido: ER and PR-negativo, HER2-positivo; Triple Negativo: ER, PR and HER2-negativo ${ }^{9}$.

\section{Análisis estadístico}

Se expresó como media y desviación estándar (DE) las variables cuantitativas, para las variables categóricas frecuencias absolutas y porcentajes, se realizaron los análisis de supervivencia usando el método de Kaplan-Meier y se midió el tiempo de supervivencia global desde la fecha del diagnóstico del cáncer, a la fecha de la última visita médica o la fecha de defunción. Los análisis estadísticos se realizaron usando STATA 12.0 (Stata Corp, TX, USA).

\section{Resultados}

Durante el periodo de estudio 4.059 pacientes fueron operadas de cáncer de seno, de estas 390 ( $9.6 \%$ ) tenían edad $\leq 40$ al momento del diagnóstico. Entre las mujeres jóvenes, 373 (95.6\%) tenían carcinoma infiltrante y 17 (4.4\%) tenían carcinoma in situ. 365 pacientes cumplieron con los criterios de inclusión, 8 fueron excluidas por no tener suficiente información en sus historias clínicas.

Las características clínicas y patológicas de las pacientes se encuentran en la Tabla 1. La edad promedio al diagnóstico fue de 35 años (DE 4.3), 328 (89.8\%) pacientes tenían cáncer ductal, 25 (6.9 \%) lobular o mixto, 187 (52.7 \%) pacientes tenían tumor con grado histológico alto, 208 (57\%) tenían ki-67 elevado ( $\geq 14 \%), 69$ (18.9\%) invasión linfovascular y 6 (1.6\%) tenían cáncer de seno bilateral.

Con respecto al estadio al diagnóstico: 67 (18.3\%) pacientes eran estadio I, 152 (41.6\%) estadio II, 140 (38.3\%) estadio III y 6 (1.6\%) estadio IV (a pesar de no ser estándar, estas pacientes fueron llevadas a cirugía por su buena respuesta a la quimioterapia sistémica). Los subtipos histológicos estuvieron repartidos así: 87 (24.1 \%) Luminal A, 152 (42.1\%) Luminal B HER2-negativo, 72 (19.9 \%) Luminal B HER2-positivo, 17 (4.7\%) HER2-enriquecido, y 33 (9.1\%) Triple Negativo. Un total de 89 (24.4\%) pacientes fueron HER2-positivas.

La mayoría de pacientes recibieron terapia multimodal, incluyendo cirugía, radioterapia y quimioterapia neo o adyuvante. $40.3 \%$ de las pacientes tuvieron cirugía conservadora, $59.7 \%$ mastectomía, $58.2 \%$ de las pacientes mastectomizadas fueron reconstruidas, y la gran mayoría de las reconstrucciones fueron hechas el día de la mastectomía (57.4\%).

A la mitad de las pacientes se les realizó ganglio centinela y de estas el $31.2 \%$ tuvieron ganglios positivos. Fue realizado vaciamiento axilar a $225(61.6 \%)$ pacientes (Tabla 1).

El $90 \%$ de las pacientes recibieron quimioterapia, la mayoría neoadyuvancia (58.9\%). La respuesta patológica completa (ypToyNo, incluido no in situ residual en ganglio ni mama) fue obtenida en el 19,2 \% de las pacientes. El régimen de quimioterapia más utilizado fue la combinación de AC y taxanes (87.8\%), seguido de solo AC. El taxane más utilizado fue el paclitaxel. 303 (83\%) 
Tabla 1. Características de los pacientes.

\begin{tabular}{|c|c|}
\hline \multirow{2}{*}{ Caracteristica } & $n=365$ \\
\hline & $\#(\%)$ \\
\hline Edad (DE) & $35(4,3)$ \\
\hline Min-Max & $20-40$ \\
\hline \multicolumn{2}{|l|}{ Histología } \\
\hline Ductal & $328(89,8)$ \\
\hline Lobular y mixto & $25(6,9)$ \\
\hline Otros $^{*}$ & $12(3,3)$ \\
\hline \multicolumn{2}{|l|}{ Grado histológico $n=355$} \\
\hline Grado 1 y 2 & $168(47,3)$ \\
\hline Grado 3 & $187(52,7)$ \\
\hline \multicolumn{2}{|l|}{ Tamaño tumoral $(\mathrm{mm}) \mathrm{n}=346$} \\
\hline$\leq 20$ & $109(31,5)$ \\
\hline $21-50$ & $159(46)$ \\
\hline$\geq 51$ & $78(22,5)$ \\
\hline \multicolumn{2}{|l|}{ Estadio al diagnóstico } \\
\hline Cáncer temprano (I-IIA) & $154(42,2)$ \\
\hline Cáncer localmente avanzado (IIB - IIIC) & $205(56,2)$ \\
\hline Metastásico (IV) & $6(1,6)$ \\
\hline \multicolumn{2}{|l|}{ Receptores hormonales (ER y/o PR positivo) } \\
\hline Positivos & $314(86,2)$ \\
\hline \multicolumn{2}{|l|}{ Her 2 neu } \\
\hline Positivo & $89(24,4)$ \\
\hline \multicolumn{2}{|l|}{ Ki-67 \% } \\
\hline$\geq 14$ & $208(57 \%)$ \\
\hline \multicolumn{2}{|l|}{ Invasión linfovascular } \\
\hline Positiva & $69(18,9)$ \\
\hline \multicolumn{2}{|l|}{ Subtipos moleculares por IHC ( $n=361)$} \\
\hline Luminal A & $87(24,1)$ \\
\hline Luminal B Her2 - & $152(42,1)$ \\
\hline Luminal B Her2 + & $72(19,9)$ \\
\hline Her2-enriquecido & $17(4,7)$ \\
\hline Triple Negativo & $33(9,1)$ \\
\hline \multicolumn{2}{|l|}{ Quimioterapia neo o adyuvante $(n=336)$} \\
\hline Neoadyuvante & $198(58,9)$ \\
\hline Adyuvante & $138(41,1)$ \\
\hline \multicolumn{2}{|l|}{ Respuesta a la quimioterapia neoadyuvante } \\
\hline Respuesta patológica completa & $38(19,2)$ \\
\hline \multicolumn{2}{|l|}{ Tipo de cirugía } \\
\hline Cuadrantectomía & $147(40,3)$ \\
\hline Mastectomía & $218(59,7)$ \\
\hline \multicolumn{2}{|l|}{ Vaciamiento axilar $\geq 10$ ganglios $(n=225)$} \\
\hline Compromiso positivo & $140(62,2)$ \\
\hline Radioterapia & $303(83,0)$ \\
\hline
\end{tabular}

recibieron radioterapia. Todas las pacientes con receptor hormonal positivo recibieron tamoxifeno y $75 \%$ de las pacientes HER-2 positivas recibieron trastuzumab.

Se le realizaron pruebas genéticas a 98 (26.8 \%) de las pacientes, 5 fueron positivas para BRCA1 y 8 para BRCA2. La prueba genética más utilizada fue la de Myriad. De las pacientes BRCA 1 y BRCA 2 mutadas, 8 fueron llevadas a mastectomía contralateral de reducción del riesgo, 2 están siendo seguidas con imágenes de seno y dos pacientes progresaron.

En 2 pacientes no se cuenta con la información de su desenlace 34 (9.3\%) de las pacientes tuvieron recaída loco-regional y 132 (36.1\%) desarrollaron metástasis a distancia. El sitio más común de metástasis fue el hueso, seguido de pulmón. 20 \% de las pacientes desarrollaron metástasis a SNC en algún momento de su enfermedad.

La mediana de seguimiento estuvo en 60 meses, la supervivencia global a cinco años fue del 81.3\% (IC95 \%: 76.3-85.4). La supervivencia global a cinco años de acuerdo al estadio fue: estadio I de $96.2 \%$, estadio II $86.4 \%$, y estadio III $73 \%$ $(\mathrm{p}<0.05)$ (Figura 1).

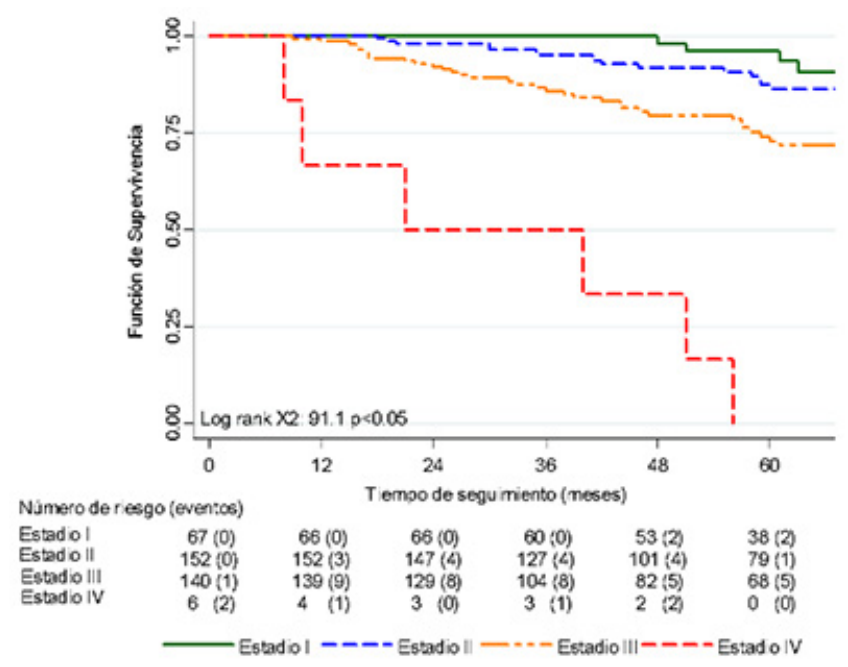

Figura 1. Supervivencia global de acuerdo al estadio.

La supervivencia global a cinco años de acuerdo a los subtipos histológicos: Luminal A $97.4 \%$ (IC95\%: 90.3-99.3), Luminal B HER2-negativo $72.8 \%$ (IC95\%: 73.3-80.3), Luminal B HER2- positivo 85.5 \% (IC95 \%: 72.7-92.6), HER2-enriquecido 74.8 \% (IC95 \%: 45.6-89.8) y Triple Negativo 67.7 \% (IC95 \%: 47.9-81.3) (Figura 2).

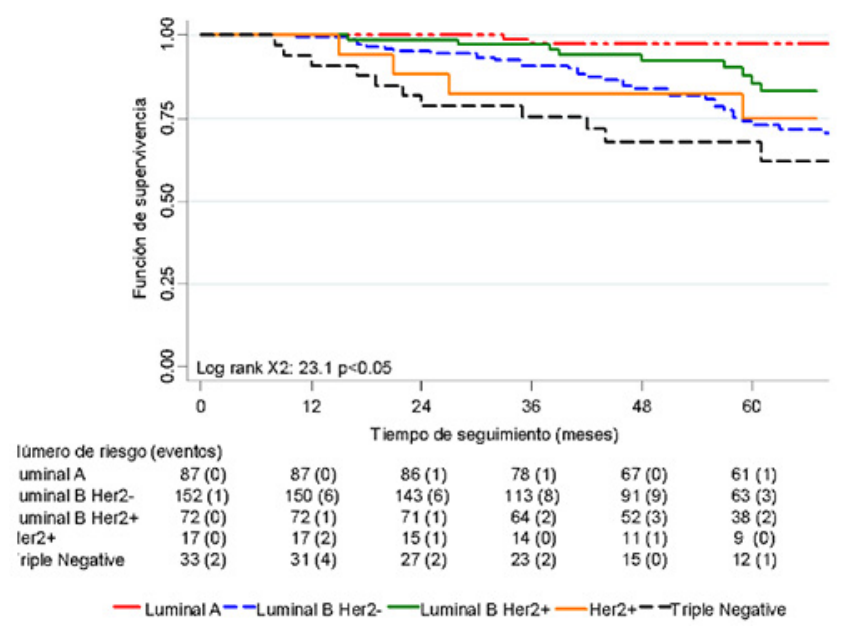

Figura 2. Supervivencia global de acuerdo a los subtipos moleculares por inmunohistoquímica. 
La mediana de tiempo o supervivencia libre de recaída (SLE) fue de 54 meses, con una SLE a cinco años de $73.7 \%$ (IC95 \%: 68.5-78.3). La SLE a cinco años según el estadio fue: estadio I 86.7 $\%$, estadio II $75.1 \%$, y estadio III $93.1 \%$ ( $p<0.05)$. La SLE de acuerdo a los subtipos histológicos: Luminal A 89.2 \% (IC95 \%: 79.5-94.4), Luminal B-HER2-negativo $68.8 \%$ (IC95 \%: 59.9-76.2), Luminal B-HER2-positivo $73.2 \%$ (IC95 \%: 60.1-82.6), HER2-enriquecido $52.3 \%$ (IC95 \%: 26.7-72.6) y triple negativo $67.6 \%$ (IC95\%: 47.9-81.1)

\section{Discusión}

En este estudio se documenta la historia natural de una cohorte de mujeres jóvenes con cáncer de seno, que recibieron tratamiento en un instituto oncológico de Colombia.

$8 \%$ de las pacientes tenían edad menor o igual a 40 años, lo cual es similar al porcentaje descrito en dos ciudades colombianas, Bucaramanga (11.6 \%) y Cali $(10.3 \%)^{3}$, pero representa una frecuencia menor comparada con otros países latinoamericanos $(20 \%)^{1,3}$, esto pudiera ser explicado por las diferencias raciales observadas en Latinoamérica y sería de interés futuro para investigación.

Con respecto a las características patológicas del tumor, hay una diferencia importante entre las pacientes colombianas y las mexicanas. Comparado con México, las pacientes colombianas tuvieron un mayor porcentaje de grado histológico alto ( $52 \%$ vs. $26 \%$ ) y un menor porcentaje de invasión linfovascular (18\% vs. $29 \%{ }^{10}$.

La distribución del cáncer con respecto a los subtipos moleculares fue diferente a la hallada en otros países latinoamericanos. Se observó un mayor porcentaje de pacientes con cáncer subtipo Luminal $B$, comparado con tipo Luminal $A$, y una mayor proporción de pacientes HER2-positivas; el porcentaje de triple negativo fue menor ${ }^{1,8}$.

El alto porcentaje de pacientes Luminal B con respecto al Luminal A fue observado en otro estudio realizado en el IDC realizado en mujeres adultas ${ }^{11}$. Países como Estados Unidos, México, Costa Rica y Perú, han descrito un porcentaje similar de tumores HER2 positivos, con una frecuencia de alrededor del 16\% 7, 10, 12,13.
En el momento del diagnóstico la mayoría de pacientes estaban en estadios II y III, esta distribución es similar a la reportada en el resto de Latinoamérica ${ }^{1,14}$. La alta incidencia de tumores en estadio III puede ser un reflejo de los problemas de acceso al sistema de salud de nuestro país, como lo menciona Piñeros en su estudio ${ }^{15}$.

El compromiso metastásico es similar al de mujeres de edad más avanzada, siendo más común el óseo. Es importante resaltar que encontramos compromiso de SNC en algún momento de la enfermedad en un $20 \%$ de las pacientes ${ }^{16}$.

El hecho de que a la mayoría de las pacientes se les realizara mastectomía, podría ser explicado por la creencia de algunos cirujanos sobre una mayor tasa de recaídas locales en mujeres jóvenes ${ }^{17}$. La edad no debería ser el único factor tomado en cuenta para tomar la decisión sobre el tipo de cirugía a realizar en estas pacientes. Se debe mirar el subtipo histológico, el estadio, los antecedentes familiares y las comorbilidades de la paciente ${ }^{18,19}$.

No se encontraron diferencias con respecto al tipo de quimioterapia recibido por las pacientes, con lo descrito en la literatura; la mayoría recibieron tanto antraciclinas como taxanes ${ }^{5,20}$. Con respecto a la hormonoterapia, todas las pacientes con tumores receptor hormonal positivo, recibieron tamoxifeno en adyuvancia. El bloqueo dual con inhibidores de GNRH no es una práctica común en la institución.

La supervivencia global a cinco años fue la esperada, de acuerdo a la revisión de la literatura (81.3\%). La SG de acuerdo a los estadios tumorales, fue similar a la reportada en el Reino Unido y en Estados Unidos ${ }^{21,22}$.

Aunque la incidencia de cáncer de seno en mujeres jóvenes de Colombia no es tan alta como en otros países latinoamericanos, debería ser considerada un problema de salud pública debido al impacto social que viven estas mujeres, ya que muchas son madres cabeza de familia ${ }^{23}$. Sería ideal contar con estrategias de tamizaje para mujeres menores de 40 años, ya que ni la mamografía ni la resonancia magnética (si la paciente tiene factores genéticos) están disponibles o no tienen cobertura para mujeres de esta edad, en el sistema de salud colombiano actual. 
La baja frecuencia de la realización de pruebas genéticas, a pesar de que se tiene disponible un servicio de consejería genética desde el 2014, se debe a problemas de cubrimiento en salud. Ha sido un reto hacer entender a las aseguradoras la importancia de un resultado genético positivo, no solo para determinar el tipo de terapia que recibe la paciente (cirugía y radioterapia), sino también para evaluar el riesgo de sus familias.

\section{Conclusión}

La principal limitación de este estudio fue la inclusión solo de pacientes que fueron llevadas a cirugía, por lo tanto, el porcentaje de pacientes con cáncer estadio IV de novo no pudo ser reportado.

Se encontró un mayor porcentaje de pacientes con cáncer Luminal B y HER2 positivo, al igual que lo reportado en la literatura. La SG fue similar a las reportadas por otros estudios y fue menor en estadios avanzados y tumores triple negativo.

- Aspectos éticos: los autores declaran que se ajusta a la Resolución 8430 de 1993, la ejecución del estudio fue aprobado y vigilado por el Comité de Ética Independiente del IDC Las Américas auna, el cual cumple con las normas de buenas prácticas clínicas en todas sus actividades.

- Fuente de financiación: los autores declaran que esta investigación no ha recibido becas específicas de agencias del sector público, sector comercial o sin ánimo de lucro.

- Conflicto de interés: los autores declaran no tener ningún conflicto de interés.

\section{Referencias}

1. Villarreal C, Aguila C, Magallanes MC, Mohar A, Bargalló E, Meneses A, et al. Breast Cancer in young women in Latin America: an unmet, growing burden. The Oncologist [Internet] 2013; 18:26-34. https://doi.org/10.1634/theoncologist.2013-0321

2. Partridge $\mathrm{AH}$, Ruddy KJ, Kennedy J, Winer EP. Model Program to improve care for a unique can- cer population: young women with Breast Cancer. J Oncol Pract [Internet] 2012;8(5):e105-10. https://dx.doi.org/10.1200\%2FJOP.2011.000501

3. Uribe C, Serrano M, Montañez G. Incidencia de cáncer de mama en mujeres menores de 40 años en el área metropolitana de Bucaramanga. Med UNAB [Internet] 2019;13(2):17-20. https://bit. ly/3mrLYGp

4. Bleyer A, O'Leary M, Barr R, Ries LAG (eds.). Cancer epidemiology in older adolescents and young adults 15 to 29 years of Age, including SEER incidence and survival: 1975-2000. National Cancer Institute, NIH Pub. No. 06-5767. Bethesda, MD 2006.

5. Ribnikar D, Ratosa I, Perhavec A, Amir E. General Overview and treatment recommendations for young women with Breast Cancer. Rev Inves Clin [Internet] 2017; 69:77-93. https://doi. org/10.24875/ric.17002175

6. Han W, Kim SW, Park AI, Kang D, Kim S-W, Youn $\mathrm{Y}-\mathrm{K}$, et al. Young age: an independent risk factor for disease-free survival in women with operable breast cancer. BMC Cancer [Internet] 2004; 4:82. https://doi.org/10.1186/1471-2407-4-82

7. Murphy, B, Day, C, Hoskin, T, Habermann, E, Boughey J. Adolescents and young adults with breast cancer have more aggressive disease and treatment than patients in their forties. Ann Surg Oncol [Internet] 2019; Nov;26(12):3920-3930. https://doi.org/10.1245/s10434-019-07653-9

8. Wolff AC, Hammond MEH, Allison K, Harvey BE, Mangu PB, Bartlett JMS, et al. Human Epidermal Growth Factor Receptor 2 testing in breast cancer: American Society of Clinical Oncology/ College of American Pathologist Clinical Practice Guidelines focused update. Arch Pathol Lab Med [Internet] 2018;142(11):1362-1364. https://doi. org/10.1200/jco.2018.77.8738

9. Park S, Koo JS, Kim MS, Park HS, Lee JS, Lee JS, et al. Characteristics and outcomes according to molecular subtypes of breast cancer as classified by a panel of four biomarkers using immunohistochemistry. Breast [Internet] 2012; 21(1):50-7. https://doi.org/10.1016/j.breast.2011.07.008

10. Robles J, Ruvalcaba E, Maffuz A, Rodríguez S. Breast cancer in Mexican women under 40. Ginecol Obstet Mex 2011;79(8): 482-8. PMID: 21966845

11. Gómez R, Ossa CA, Montoya ME, Echeverri C, Án- 
gel G, Ascuntar J, et al. Impact of inmunohistochemistry-based molecular subtype on chemosensitivity and survival in Hispanic breast cancer patients following neoadjuvant chemotherapy. Ecancermedicalsciencie [Internet] 2015; 9: 562. https://dx.doi.org/10.3332\%2Fecancer.2015.562

12. Quirós JL, Arce IS, Ramírez B. Cáncer de mama en mujeres jóvenes características clínicas y patológicas. Rev Med Univ Costa Rica [Internet] 2006;4(2):54-64. https://doi.org/10.15517/rmu. v4i2.7881

13. Vallejos C, Gómez HL, Abugattas JE, Marcelo MJ, Doimi FF, Neciosup SP, et al. Clinicpathologic, molecular subtype, and survival prognostic features in premenopausal breast cancer patients by age at diagnosis. ASCO Meeting Abstracts [Internet] 2010;28(suppl 15):653. https:// doi.org/10.1200/jco.2010.28.15_suppl.653

14. Assi HA, Khoury KE, Dbouk H, Khalil LE, Mouhieddine TH, El Saghir NS. Epidemiology and prognosis of breast cancer in young women. J Thorac Dis [Internet] 2013;5(Suppl 1): S2-8. https://doi.org/10.3978/j.issn.2072-1439.2013.05.24

15. Piñeros M, Sánchez R, Perry F, García OA, Ocampo R, Cendales R. Demoras en el diagnóstico y tratamiento de mujeres con cáncer de mama en Bogotá, Colombia. Salud Pública México [Internet] 2011;53(6):478-85. https://www. redalyc.org/articulo.oa?id=10621567003

16. Johnson RH, Chien FL, Bleyer A. Incidence of Breast Cancer with distant involvement among women in the United States, 1976 to 2009. JAMA [Internet] 2013;309(8):800-5. https://doi. org/10.1001/jama.2013.776

17. Paluch S, Pagani O, Partridge AH, Bar-Meir E, Fallowfield L, Fenlon D, et al. Second International Consensus Guidelines for Breast Cancer in young women $\left(\mathrm{BCY}_{2}\right)$. The Breast [Inter- net] 2016; 26:87-99. https://doi.org/10.1016/j. breast.2015.12.010

18. Cardoso F, Loibl S, Pagani O, Graziottin A, Panizza P, Martincich L, et al. The European Society of Breast Cancer Specialists Recommendations for the Management of Young Women with Breast Cancer. Eur J Cancer [Internet] 2012;48(18):335577. https://doi.org/10.1016/j.ejca.2012.10.004

19. Paluch S, Pagani O, Partridge $A H$, Abulkhair O, Cardoso M-J, Dent RA, et al. ESO-ESMO 3rd International Consensus Guidelines for Breast Cancer in Young Women ( $\left.\mathrm{BCY}_{3}\right)$. The Breast [Internet] 2017; 35:203-17. https://doi.org/10.1016/j. breast.2017.07.017

20. Early Breast Cancer Trialists' Collaborative Group (EBCTCG). Long-term outcomes for neoadjuvant versus adjuvant chemotherapy in early breast cancer: meta-analysis of individual patient data from ten randomised trials. Lancet Oncol [Internet] 2018;19(1):27-39. https://doi. org/10.1016/S1470-2045(17)30777-5

21. Xiong Q, Valero V, Kau V, Kau SW, Taylor S, Smith TL, et al. Female patients with breast carcinoma age 30 years and younger have a poor prognosis: the M.D. Anderson Cancer Center experience. Cancer [Internet] 2001;92(10):2523-8. https://doi.org/10.1002/1097-0142(20011115)92:10\%3C2523::aid-cncr1603\%3E3.0.co;2-6

22. Nixon, A, Neuberg, D, Daniel, H, Gelman, R. Relationship of patient age to pathologic features of the tumor and prognosis for patients with stage I or II breast cancer. Journal of Clinical Oncology [Internet] 1994;12(5):888-94. https://doi. org/10.1200/jco.1994.12.5.888

23. Romero, J, Angarita, M, Burbano, R. Tamizaje del cáncer de seno de acuerdo a la experiencia médica. Rev Colomb Cirugía 2014;18(1). 